\title{
Coexistence of Juvenile Systemic Lupus Erythematosus and Juvenile Spondyloarthropathy: A Case Report and Review of the Literature
}

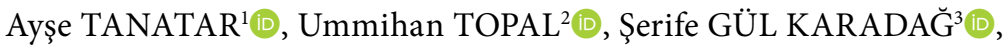 \\ Hafize Emine SÖNMEZ ${ }^{3}$ (D), Nuray AKTAY AYAZ ${ }^{3}$ (D) \\ ${ }^{1}$ Department of Pediatric Rheumatology, Istanbul University, Istanbul Faculty of Medicine, Istanbul, Turkey \\ ${ }^{2}$ Department of Radiology, University of Health Science, Kanuni Sultan Süleyman Training and Research Hospital, Istanbul, Turkey \\ ${ }^{3}$ Department of Pediatric Rheumatology, University of Health Science, Kanuni Sultan Süleyman Training and Research Hospital, Istanbul, Turkey
}

\begin{abstract}
Sacroiliitis has been scarcely reported in patients with systemic lupus erythematosus (SLE). In this article, we presented a pediatric case with coexistence of juvenile SLE and juvenile spondyloarthropathy (SpA) and discussed the clinical and laboratory findings by the literature review. A 16-year-old female patient with a diagnosis of SLE was referred to our outpatient clinic with inflammatory low-back pain for two months. Sacroiliac magnetic resonance imaging confirmed the presence of bilateral active sacroiliitis. She was finally diagnosed with juvenile SpA. She achieved remission with subcutaneous methotrexate and non-steroid anti-inflammatory drugs. To the best of our knowledge, this is the first pediatric case with SLE and SpA. This case provides further implication about atypical presentation of a well-known disorder.

Keywords: : Juvenile spondyloarthropathy, sacroiliitis, systemic lupus erythematosus.
\end{abstract}

Systemic lupus erythematosus (SLE) is an autoimmune disease that involves multiple organ systems. Musculoskeletal (MS) involvement occurs in either during the disease course in $70-95 \%$ of SLE patients or as an initial finding in nearly 50\% of the cases. ${ }^{1,2}$ MS involvement may vary from myalgia, arthralgia, non-erosive arthritis, myositis, tenosynovitis to contractures and avascular necrosis. ${ }^{2}$ However, co-existence of sacroiliitis ${ }^{3,4}$ or spondyloarthropathy $(\mathrm{SpA})$ is really scarce..$^{5}$ In this article, we reported a patient presenting with juvenile SLE and SpA and discussed the clinical and laboratory findings by the literature review. To the best of our knowledge, this is the first pediatric case with coexistence of juvenile SLE and SpA.

\section{CASE REPORT}

A 16-year-old Syrian female patient was admitted to our hospital with low-back pain present for two months. She was born by vaginal delivery at 39 weeks as the second child of consanguineous parents (first cousins). She had initially presented to a local medical center with fever, rash, fatigue, oral ulcers and pancytopenia a year before. She was diagnosed with SLE. Prednisolone $(1 \mathrm{mg} / \mathrm{kg})$ and hydroxychloroquine therapy was administered. One year later, she was referred to our hospital due to complaints of low-back pain worsening in the morning and after a long resting period during the last two months. She also suffered from morning stiffness lasting two-three hours and heel pain. On physical

Received: February 25, 2019 Accepted: May 21, 2019 Published online: January 08, 2020

Correspondence: Nuray Aktay Ayaz, MD. SBÜ Kanuni Sultan Süleyman Eğitim ve Araştırma Hastanesi Çocuk Romatoloji Kliniği, 34303 Küçükçekmece, İstanbul, Turkey. Tel: +90 532 - 2765238 e-mail: nurayaktay@gmail.com 
examination, Flexion, ABduction, External Rotation test and sacroiliac compression tests were all positive. She had tenderness in sacroiliac joints (SIJs) and heels. Schober test result was $5 \mathrm{~cm}$. With these findings, she was hospitalized for a detailed evaluation. In laboratory work-up, baseline complete blood count, biochemical tests, and complete urinalysis were normal. Erythrocyte sedimentation rate was $43 \mathrm{~mm} /$ hour and C-reactive protein was $9.4 \mathrm{mg} / \mathrm{L}$. The homogenous nucleolar antinuclear antibody pattern was found to be positive at $1 / 320$ titer. Anti-double stranded deoxyribonucleic acid was also positive. Anticardiolipin and anti- $\beta 2$ glycoprotein antibodies, lupus anticoagulant were all negative. She had normal levels of complement 3 and 4 . She underwent sacroiliac and thoracolumbar magnetic resonance imagining (MRI). Sacroiliac MRI revealed active sacroiliitis in the right side based upon bone marrow edema in short tau inversion recovery sequences and increased enhancement in post contrast sequences (Figure 1a). However, thoracolumbar MRI was normal. Human leukocyte antigen (HLA)-B27 was negative. Since she used prednisolone during one year period without any calcium replacement, dual energy X-ray absorptiometry (DXA) was performed. DXA showed L2-L4 Z-score: -2.6 standard deviation (SD), neck of hip Z-score: -2.5 SD. Anti-resorptive treatment including pamidronate, calcium and vitamin $\mathrm{D}$ were administered. Steroid treatment was tapered to $5 \mathrm{mg}$ per day. She had a low socio-cultural level and used immunosuppressive agents approximately for one year. Therefore, she was screened for presence of tuberculosis (TB). Tuberculin skin test was $0 \mathrm{~mm}$ and computed tomography (CT) of the thorax was normal. Finally, CT-guided needle biopsy of the right SIJ was performed and histopathological examination revealed chronic inflammation (Figure 1b). Bacterial and TB cultures, as well as TB polymerase chain reaction were all negative. She was diagnosed with juvenile SpA after exact exclusion of differential diagnoses. Subcutaneous methotrexate (15 mg/m²/weekly), folic acid supplement, and non-steroid anti-inflammatory drug were started. Her symptoms responded to this treatment positively. A written informed consent was obtained from the legal guardians of the patient.

We conducted a systematic review of the published literature about SLE and SpA. We searched the literature from inception to January 2019, using the following keywords; 'systemic lupus erythematosus', 'SLE', 'juvenile systemic lupus erythematosus', 'juvenile SLE', 'spondyloarthropathy', 'sacroiliitis', 'ankylosing spondylitis' and 'pediatric'. Both searches were limited to English language. Two investigators
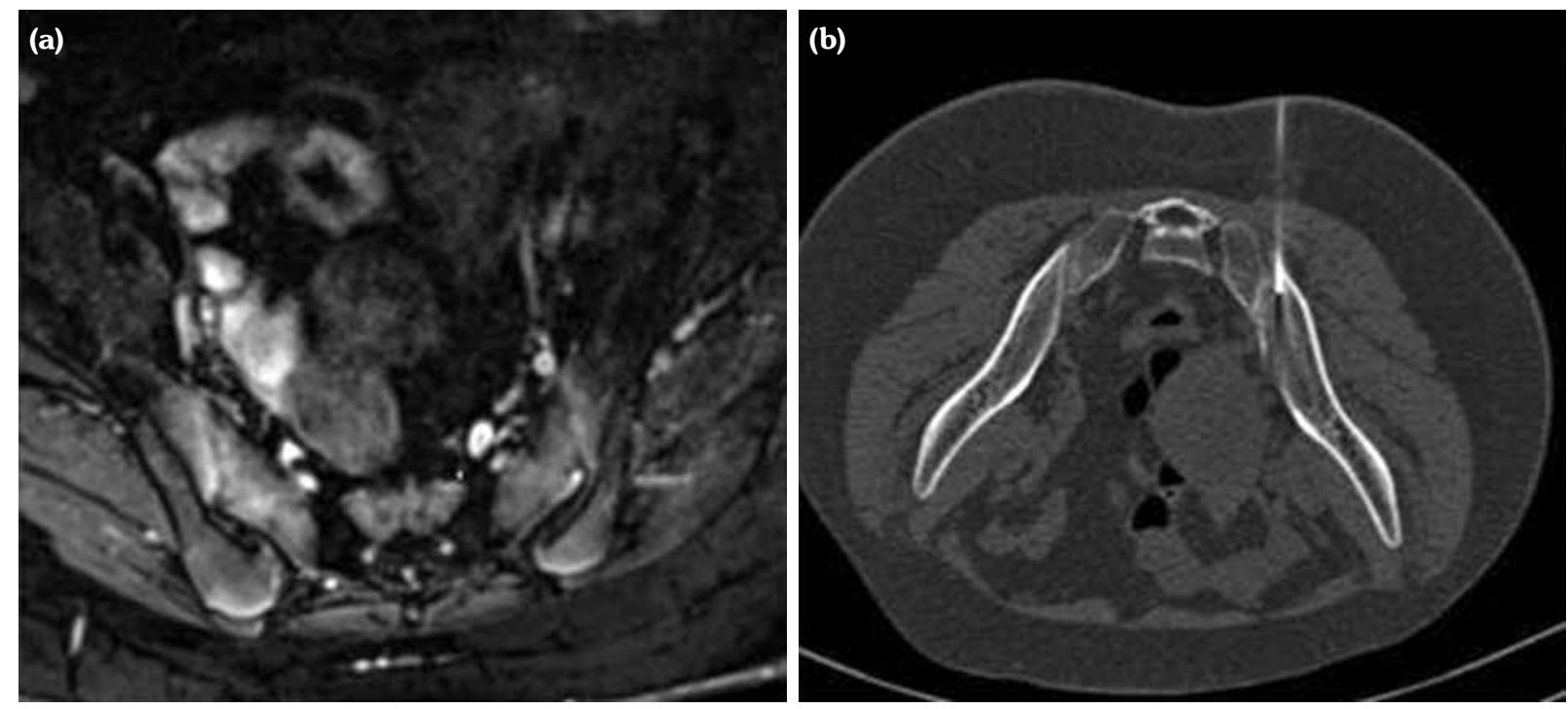

Figure 1. (a) Sacroiliac magnetic resonance imagining of patient with juvenile systemic lupus erythematosus and spondyloarthropathy (active sacroiliitis in right side). (b) Computed tomography-guided needle biopsy of right sacroiliac joint. 


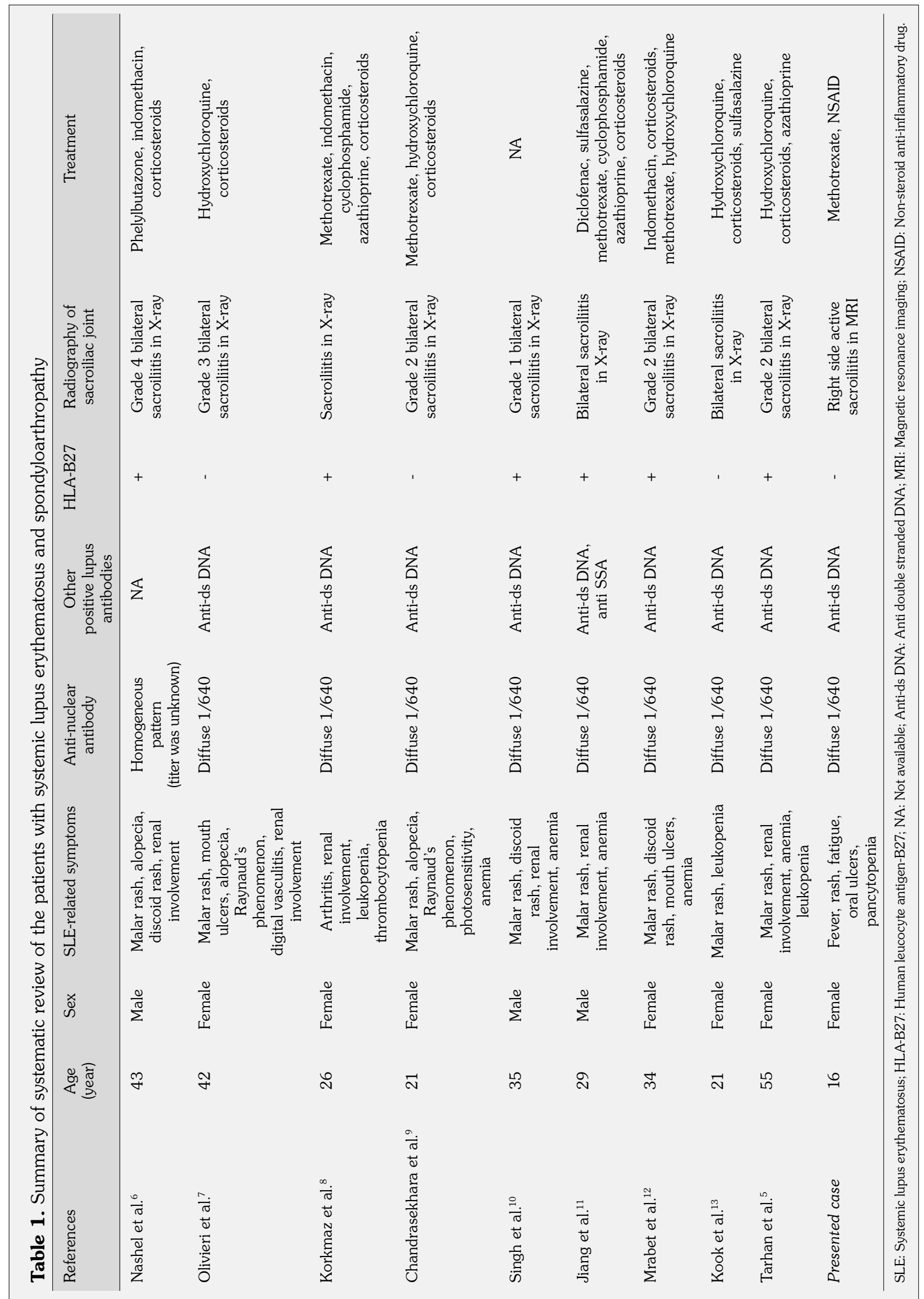


independently reviewed potentially eligible articles. Discrepancies were resolved by discussion between the authors and with a third author. Up to date, only 10 adult patients with SLE and SpA have been described (Table 1). ${ }^{5-13}$

\section{DISCUSSION}

The co-existence of SLE and SpA is rare. The presented patient was the first pediatric case in the literature. Previously, 10 adult patients with SLE and SpA have been reported (Table 1).5-13 Among them, eight patients (80\%) had malar rash, seven (70\%) had hematologic involvement, four (40\%) had renal involvement and three (30\%) had discoid rash revealing SLE. ${ }^{5-13}$ All patients fulfilled the American Collage of Disease criteria for SLE. ${ }^{14}$ Furthermore, six patients had peripheral arthritis. HLA-B27 was positive in six patients. The X-ray findings revealed sacroiliitis in all of them and all patients were classified as having SpA according to Modified New York Criteria for ankylosing spondylitis (AS). ${ }^{15}$ Our patient also fulfilled the previously described pediatric criteria for these two diseases. ${ }^{16,17}$ However, she was negative for HLA-B27.

Systemic lupus erythematosus patients are prone to develop common and opportunistic infections like TB due to intrinsic immunologic defects and immunosuppressive therapy introduced. ${ }^{18}$ Patients with SLE had increased incidence of $\mathrm{TB}$ compared to the general population and TB of the SIJ in SLE has been previously reported. ${ }^{18}$ Since our patient had a low socio-cultural level and used immunosuppressive agents for more than a year, we first focused to evaluate the presence of TB. However, TB was excluded by radiologic, laboratory and histopathological examinations.

Joint involvement in SLE is typically symmetric, non-erosive and non-deforming, with polyarticular involvement, affecting both large and small joints. Although it is rare, isolated sacroiliitis have been previously described in patients with SLE. ${ }^{3,4}$ Juvenile SpA is a group of seronegative arthritis which is characterized by enthesitis, asymmetrical oligoarthritis of lower extremities, axial involvement and HLA B27 positivity and starts prior to 16 years of age. ${ }^{17}$ It is difficult to distinguish whether sacroiliitis is a musculoskeletal finding of SLE or if it is due to the coexistence of two diseases; SLE and SpA. The presented case met the criteria for juvenile SpA with presence of sacroiliitis and enthesitis. However, she was negative for HLA-B27 antigen. Different pathogenetic mechanisms play role in these two diseases, but combination of the susceptibility genes or epigenetic factors may lead to this rare coexistence. For instance, previous studies have demonstrated that microRNA-16, a small noncoding ribonucleic acid molecule displaying role in development of autoimmune disease, is down-regulated in patients with SLE and AS. ${ }^{19}$ However, to date, immunogenetic mechanisms underlying the coexistence of SLE and SpA are still unclear. We need more evidence to speculate about these pathogenic mechanisms and this case may point out to the need for further approaches about this rare association.

In conclusion, we described a pediatric patient with concomitant SLE and SpA. However, this coexistence is rare and improvement of the knowledge on these diseases may guide us to define the underlying pathogenesis. Further multicenter studies with more patients and prospective follow-up are required to understand the whole spectrum of these diseases.

\section{Declaration of conflicting interests}

The authors declared no conflicts of interest with respect to the authorship and/or publication of this article.

\section{Funding}

The authors received no financial support for the research and/or authorship of this article.

\section{REFERENCES}

1. Ball EM, Bell AL. Lupus arthritis--do we have a clinically useful classification? Rheumatology (Oxford) 2012;51:771-9.

2. Zoma A. Musculoskeletal involvement in systemic lupus erythematosus. Lupus. 2004;13:851-3.

3. Lee SS. Symptomatic unilateral sacroiliitis in systemic lupus erythematosus. Lupus. 1995;4:328-9.

4. Kohli M, Bennett RM. Sacroiliitis in systemic lupus erythematosus. J Rheumatol 1994;21:170-1.

5. Tarhan F, Argın M, Can G, Özmen M, Keser G. Coexistence of systemic lupus erythematosus and ankylosing spondylitis: another case report 
and review of the literature. Eur $\mathrm{J}$ Rheumatol 2014;1:39-43.

6. Nashel DJ, Leonard A, Mann DL, Guccion JG, Katz AL, Sliwinski AJ. Ankylosing spondylitis and systemic lupus erythematosus: a rare HLA combination. Arch Intern Med 1982;142:1227-8.

7. Olivieri I, Gemignani G, Pasero G. Ankylosing spondylitis with exuberant sclerosis in the sacroiliac joints, symphysis pubis and spine. $\mathrm{J}$ Rheumatol 1990;17:1515-9.

8. Korkmaz C. Delayed diagnosis of porphyria based on manifestations of systemic lupus erythematosus and ankylosing spondylitis. J Nephrol 2006;19:535-9.

9. Chandrasekhara PK, Jayachandran NV, Thomas J, Narsimulu G. Systemic lupus erythematosus and dermatomyositis with symptomatic bilateral sacroiliitis: an unusual and interesting association. Mod Rheumatol 2009;19:84-6.

10. Singh S, Sonkar GK, Singh U. Coexistence of ankylosing spondylitis and systemic lupus erythematosus. J Chin Med Assoc 2010;73:260-1.

11. Jiang L, Dai X, Liu J, Ma L, Yu F. Hypoparathyroidism in a patient with systemic lupus erythematosus coexisted with ankylosing spondylitis: a case report and review of literature. Joint Bone Spine 2010;77:608-10.

12. Mrabet D, Rekik S, Sahli H, Trojet S, Cheour I, Eleuch $\mathrm{M}$, et al. Ankylosing spondylitis in female systemic lupus erythematosus: a rare combination. Lupus 2011;20:777-8.
13. Kook MH, Yoo HG, Hong MJ, Yoo WH. Coexisting systemic lupus erythematosus and ankylosing spondylitis: a case report and review of the literature. Lupus 2012;21:348-9.

14. Hochberg MC. Updating the American College of Rheumatology revised criteria for the classification of systemic lupus erythematosus. Arthritis Rheum 1997;40:1725.

15. van der Linden S, Valkenburg HA, Cats A. Evaluation of diagnostic criteria for ankylosing spondylitis. A proposal for modification of the New York criteria. Arthritis Rheum 1984;27:361-8.

16. Petri M, Orbai AM, Alarcón GS, Gordon C, Merrill JT, Fortin PR, et al. Derivation and validation of the Systemic Lupus International Collaborating Clinics classification criteria for systemic lupus erythematosus. Arthritis Rheum 2012;64:2677-86.

17. Petty RE, Southwood TR, Manners P, Baum J, Glass $\mathrm{DN}$, Goldenberg $\mathrm{J}$, et al. International League of Associations for Rheumatology classification of juvenile idiopathic arthritis: second revision, Edmonton, 2001. J Rheumatol 2004;31:390-2.

18. Erdozain JG, Ruiz-Irastorza G, Egurbide MV, Martinez-Berriotxoa A, Aguirre C. High risk of tuberculosis in systemic lupus erythematosus? Lupus 2006;15:232-5.

19. Yan L, Liang M, Hou X, Zhang Y, Zhang H, Guo Z, et al. The role of microRNA-16 in the pathogenesis of autoimmune diseases: A comprehensive review. Biomed Pharmacother 2019;112:108583. 